\title{
A parallel implementation of the ghost-cell immersed boundary method with application to stationary and moving boundary problems
}

\author{
S PETER and A K DE* \\ Department of Mechanical Engineering, Indian Institute of Technology Guwahati, Guwahati, Assam 781039, \\ India \\ e-mail: akd@iitg.ernet.in
}

MS received 1 January 2015; revised 7 August 2015; accepted 12 November 2015

\begin{abstract}
A modified version of the previously reported ghost-cell immersed boundary method is implemented in parallel environment based on distributed memory allocation. Reconstruction of the flow variables is carried out by the inverse distance weighting technique. Implementation of the normal pressure gradient on the immersed surface is demonstrated. Finite volume method with non-staggered arrangement of variables on a nonuniform cartesian grid is employed to solve the fluid flow equations. The proposed method shows reasonable agreement with the reported results for flow past a stationary sphere, rotating and transversely oscillating circular cylinder.
\end{abstract}

Keywords. Taylor series; inverse distance weighting; Neumann boundary condition; parallelization; moving boundary.

\section{Introduction}

The physics of flows involving complex geometries found in nature and engineering applications is complicated. Conventionally, complex geometries are handled using body-fitted structured or unstructured grids. Structured grid with finite difference discretization requires coordinate transformation leading to computation of extra geometric coefficients. The unstructured grid, on the other hand, has an advantage of more controlled local mesh refinement than the structured grid. However, the grid generation, data structure and formulations are highly involved in these conventional routes. Difficulties of handling spatial terms in the body-conformal grids and simplicity of the cartesian grids have motivated to adopt non-body conformal cartesian grids for simulating complex flows.

Immersed boundary method (IBM) was first introduced by Peskin [1], for boundaries that interact with the surrounding fluid. He performed two-dimensional simulations of flow through the natural mitral heart valve on uniform cartesian grid. Sensitization of the immersed boundary to a non-conformal grid was accomplished by a semi-discrete analog of the Dirac delta function. Numerically this treatment is suitable for flexible elastic boundaries, but its implementation on rigid bodies leads to stiffness issues. Goldstein et al [2] proposed a feedback forcing approach that required two ad-hoc user specified parameters, for imposing boundary conditions on rigid bodies. Both these

*For correspondence approaches required spreading of the forcing around the immersed boundary, hence are hardly justified for high Reynolds number flows.

Mohd-Yosuf [3] proposed a discrete time forcing approach that does not require spreading of the forcing. Moreover, for unsteady flows this approach does not severely limit the time step as in the previous approaches. Kim et al [4] accounted for the possible mass source/sink in the Mohd-Yosuf [3]'s approach. A ghost-cell immersed boundary method (GCIBM), which imposes the boundary condition by an interpolation of the variables at the ghostcell, was proposed by Majumdar et al [5]. In this approach the computational cells are identified as fluid (or solid) cells if they are located outside (or inside) the complex geometry, respectively. Solid cells having at least one fluid cell in their neighborhood are then identified as the ghost-cells.

The incorporation of various boundary conditions (Dirichlet, Neumann and Robin) in GCIBM was demonstrated by Tseng and Ferziger [6]. Mittal et al [7] and De [8] extended this approach to problems involving highly complex three-dimensional and moving boundaries. Lee and You [9] coupled GCIBM with the mass source/sink algorithm of Kim et al [4], for controlling the production of spurious force oscillations, which is a severe issue in simulating moving boundary problems using IBM in the inertial frame of reference. Kim and Choi [10] developed an IBM in the noninertial frame of reference to solve the flow around an arbitrarily moving boundary with complex geometry.

GCIBM involves matrix inversion, thereby strongly depends upon the distribution of the computing cells. To 
circumvent this issue Iaccarino and Verzicco [11] suggested the use of inverse distance weighting (IDW), while Gao et al [12] demonstrated the use of Taylor series expansion coupled with IDW for reconstruction of variables at the ghost-cell. An extensive review on the developments, usage and implications of IBM can be found in the article of Mittal and Iaccarino [13].

There are numerous advantages of IBM, such as ease of grid generation, incorporating body motions, CPU memory and time savings, ease of implementing fast and stable iterative linear solvers and amenability to geometric multi-grid methods. However, systematic mesh refinement and enforcing boundary conditions on non-conformal grids are two major issues with the method which are yet not fully resolved. Objective of this work is to enhance the capabilities of the GCIBM which was formulated by Gao et al [12].

\section{Parallelized GCIBM for moving boundary problems}

The normalized (using $D, U$ and $D / U$ as length, velocity and time scales, respectively) governing equations for incompressible flow with constant fluid properties in the non-inertial frame of reference [14] are

$$
\begin{gathered}
\frac{\partial u_{i}}{\partial x_{i}}=0 \\
\frac{\partial u_{i}}{\partial t}+\frac{\partial\left(u_{i} u_{j}\right)}{\partial x_{j}}=-\frac{\partial p}{\partial x_{i}}+\frac{1}{R e} \frac{\partial^{2} u_{i}}{\partial x_{j} \partial x_{j}}-a_{i}
\end{gathered}
$$

where $R e=U D / v$ is the Reynolds number and $a_{i}$ is $i$ th component of the acceleration of a body with respect to the inertial frame of reference.

\subsection{Taylor series and IDW reconstruction for $G C I B M$}

Figure 1 shows a typical near boundary stencil which comprises a ghost-cell $(G)$, immediate neighboring fluid cells $(F)$ and boundary point $(B)$. The boundary (or forcing) point is so chosen that $\overrightarrow{G B}$ points in the surface normal direction at $B$. The fluid neighbors $F_{1}, F_{2}, F_{3}, F_{4}$ $\ldots F_{n}, F_{n+1}$ are at distances $h_{1}<h_{2}<h_{3}<h_{4} \ldots h_{n}<R$ from $B$. Instead of fitting a localized polynomial [5-9], Taylor series expansion about $B$ can serve to reconstruct [12] the solution at the ghost point

$$
\begin{aligned}
\phi_{G}= & \phi_{B}+\Delta x_{i}\left(\frac{\partial \phi}{\partial x_{i}}\right)_{B}+\frac{1}{2} \Delta x_{i} \Delta x_{j}\left(\frac{\partial^{2} \phi}{\partial x_{i} \partial x_{j}}\right)_{B} \\
& +3^{\text {rd }} \text {-order terms }
\end{aligned}
$$

where $i, j$ expand for spatial directions with $\Delta x_{i}=x_{i, G}-$ $x_{i, B}(i=x, y, z)$.

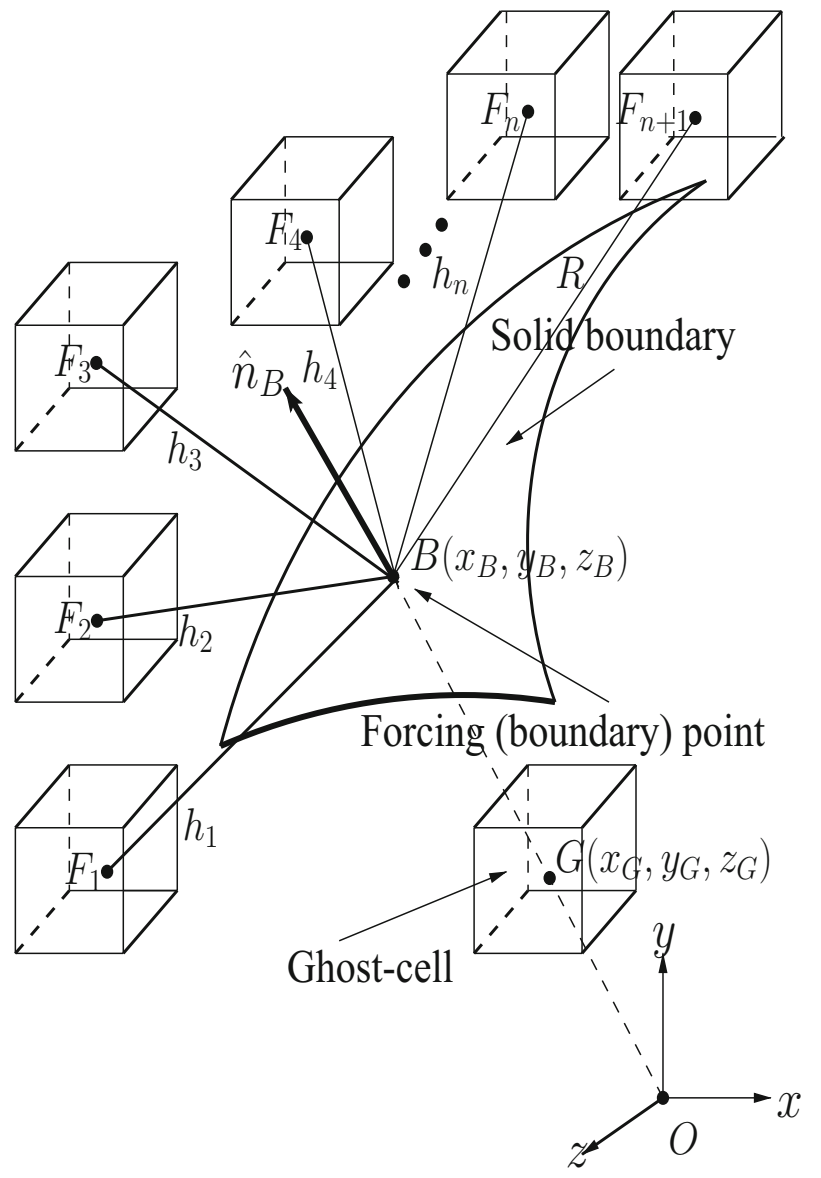

Figure 1. Schematic diagram for evaluating flow variables at the ghost-cell centroid.

At the boundary points, IDW [12] interpolation can be used for the flow variables and their partial derivatives

$$
\begin{aligned}
\phi_{B} & =\frac{1}{q} \sum_{m=1}^{n} w_{m} \phi_{m},\left(\frac{\partial \phi}{\partial x_{i}}\right)_{B}=\frac{1}{q} \sum_{m=1}^{n} w_{m}\left(\frac{\partial \phi}{\partial x_{i}}\right)_{m},\left(\frac{\partial^{2} \phi}{\partial x_{i} \partial x_{j}}\right)_{B} \\
& =\frac{1}{q} \sum_{m=1}^{n} w_{m}\left(\frac{\partial^{2} \phi}{\partial x_{i} \partial x_{j}}\right)_{m}
\end{aligned}
$$

where $w_{m}=\left(1 / h_{m}-1 / R\right)^{2}$ are the weighting coefficients and $q=\sum_{m=1}^{n} w_{m}$. All the derivatives at the fluid points are calculated (see Appendix) on a non-uniform grid with the latest available values of the flow variables; instead of previous time step values on a uniform grid [12]. It has been observed that $n=4-5$ (excluding the one with distance $R$ ) are enough to enforce the boundary conditions on the immersed surface, which is in agreement with Gao et al [12].

Dirichlet boundary condition: A specified value for a variable $\phi_{D}$ implies

$$
\phi_{B}=\phi_{D}
$$

Thus the ghost point value can be evaluated using Eqs. (3), (4) and (5). 
Neumann boundary condition: If $\hat{n}$ is the unit normal vector $\left(n_{x}, n_{y}, n_{z}\right)$ at $B$, a specified normal gradient for a variable $\left(\frac{\partial \phi}{\partial n}\right)_{N}$ implies

$$
(\nabla \phi)_{B} \cdot \hat{n}_{B}=\left(\frac{\partial \phi}{\partial n}\right)_{N} .
$$

This condition simplifies to

$$
\begin{gathered}
\left(n_{x} \frac{\partial \phi}{\partial x}\right)_{B}+\left(n_{y} \frac{\partial \phi}{\partial y}\right)_{B}+\left(n_{z} \frac{\partial \phi}{\partial z}\right)_{B}=\left(\frac{\partial \phi}{\partial n}\right)_{N} \\
\Longrightarrow\left(\frac{\partial \phi}{\partial z}\right)_{B}=\frac{\left(\frac{\partial \phi}{\partial n}\right)_{N}-\left(n_{x} \frac{\partial \phi}{\partial x}\right)_{B}-\left(n_{y} \frac{\partial \phi}{\partial y}\right)_{B}}{\left(n_{z}\right)_{B}} .
\end{gathered}
$$

Using the chain rule of partial differentiation, we can write

$$
\left(\frac{\partial^{2} \phi}{\partial x_{i} \partial z}\right)_{B}=\left(\frac{\partial}{\partial x_{i}}\left\{\frac{\partial \phi}{\partial z}\right\}\right)_{B}
$$

In case of planar object $\left(n_{z}=0\right)$, either $\frac{\partial \phi}{\partial x}$ or $\frac{\partial \phi}{\partial y}$ replaces $\frac{\partial \phi}{\partial z}$ in Eq. (7), thereby making it applicable for any geometry with an implicit analytical description of the surface.

\subsection{Challenges in parallelization of GCIBM}

Implementation of GCIBM in a distributed memory based parallel environment is a challenging task. If a ghost-cell is near to an interface of a processor then the ghost-cell and the corresponding fluid neighbors (on the basis of sorting) may fall in different processors. In such a situation the interpolation may fail because of no information about the neighboring fluid cells. To overcome this difficulty there are two approaches: (1) restrict the search for fluid neighbor to the host processor, (2) communicate the information of the fluid neighbors to the host processor. The second approach puts an overhead of complex communications, so we have adopted the first approach.

Domain decomposition causes the ghost-cells to distribute non-uniformly among various processors. This non-uniformity adversely affects the speed-up and efficiency of the parallel computations. Some processors may have more computing load because of more number of ghost-cells. The variation in the distribution of the ghost-cells may also lead to stall or blowing up of the computations, especially if the first approach discussed in the previous paragraph is adopted.

\subsection{Application of GCIBM to moving boundary problems}

Flow past an accelerating body can be solved in inertial or non-inertial frames of reference. In the inertial frame of reference, as the body moves all geometrical constructions need to be recomputed at every time step which slows the calculations. Moreover, since the body and thus the associated ghost-cells move frequently, the above-mentioned issue of complex communications takes the center stage. Also, the application of IBM to moving boundary problems in the inertial frame of reference encounters spurious force oscillation. The use of non-inertial frame avoids these difficulties as the body remains stationary, and only the boundary moves which is accommodated in the boundary conditions.

\subsection{Enhanced capabilities of the modified GCIBM}

Use of non-uniform grids near solid walls with parallelization is essential for simulating large scale 3D problems. Moreover, imposing zero-normal pressure gradient on the surface of an immersed boundary is crucial in obtaining a physically correct flow field. Both these features are added in the current development which is demonstrated by test cases. This renders the modified GCIBM even more robust and versatile.

The use of IDW technique near an immersed surface breaks the banded structure of the resulting matrix. This issue was handled in Gao et al [12] by taking all the supporting point values from the previous time step. However, in the modified GCIBM technique the latest available values are used which makes the convergence rate faster; and now the supporting points which are responsible for enforcing boundary conditions on an immersed boundary are strongly coupled.

The treatment of non-inertial frame is also added in the modified GCIBM-IDW method to deal with complex moving boundary problems in three dimension. It is expected that all the additions implemented in the modified GCIBM-IDW method enhance the capabilities of the existing technique.

\section{Numerical approximations and solution strategy}

The normalized governing equations are solved using the finite volume method with non-staggered arrangement of variables on a non-uniform cartesian grid. Time integration of Eq. (2) is carried out by the $2^{\text {nd }}$-order accurate Adams Bashforth Crank-Nicholson (ABCN) scheme. All the spatial terms are approximated using $2^{\text {nd }}$-order accurate schemes. Using the above-mentioned techniques Eqs. (1) and (2) take the following discrete forms, respectively

$$
\sum_{f} F_{f}^{n+1}=0
$$

$$
\begin{aligned}
& \frac{u_{i, P}^{n+1}-u_{i, P}^{n}}{\Delta t} V_{P}+\frac{1}{2} \sum_{f}\left(3 F_{f}^{n} u_{i, f}^{n}-F_{f}^{n-1} u_{i, f}^{n-1}\right) \\
& =-\frac{1}{2} \sum_{f}\left(p_{f}^{n+1}+p_{f}^{n}\right) S_{f, i}+\frac{1}{2 R e} \sum_{f}\left(F_{d f u_{i}}^{n+1}+F_{d f u_{i}}^{n}\right) \\
& \quad-\frac{V_{P}}{2}\left(a_{i}{ }^{n+1}+a_{i}{ }^{n}\right)
\end{aligned}
$$


where $V_{P}$ is the volume of a typical finite volume with centroid as $P$ having faces $f$ through which mass flux is $F_{f}$. The diffusion flux of a scalar $\phi$ is approximated as

$$
\begin{aligned}
\int_{V} \frac{\partial^{2} \phi}{\partial x_{j} \partial x_{j}} \mathrm{~d} V & =\int_{V} \nabla \cdot \nabla \phi \mathrm{d} V \\
& =\int_{S} \nabla \phi \cdot \mathbf{d} \boldsymbol{S} \approx \sum_{f}(\nabla \phi)_{f} \cdot \boldsymbol{S}_{f} \approx \sum_{f} F_{\mathrm{d} f \phi} .
\end{aligned}
$$

\subsection{Velocity-pressure coupling}

A provisional velocity $\left(\boldsymbol{u}_{\boldsymbol{P}}^{*}\right)$ excluding the pressure term of Eq. (9) is

$$
\begin{aligned}
& \frac{u_{i, P}^{*}-u_{i, P}^{n}}{\Delta t} V_{P}+\frac{1}{2} \sum_{f}\left(3 F_{f}^{n} u_{i, f}^{n}-F_{f}^{n-1} u_{i, f}^{n-1}\right) \\
& =\frac{1}{2 \operatorname{Re}} \sum_{f}\left(F_{d f u_{i}}^{*}+F_{d f u_{i}}^{n}\right)-\frac{V_{P}}{2}\left(a_{i}{ }^{n+1}+a_{i}{ }^{n}\right) .
\end{aligned}
$$

The provisional face velocity $\left(\boldsymbol{u}_{\boldsymbol{f}}^{*}=L\left\{\boldsymbol{u}_{\boldsymbol{P}}^{*}, \boldsymbol{u}_{\boldsymbol{n} \boldsymbol{b}}^{*}\right\}\right)$, where $L$ represents a linear interpolation between the velocity at a cell centroid $\left(\boldsymbol{u}_{\boldsymbol{P}}^{*}\right)$ and neighboring cell centroids $\left(\boldsymbol{u}_{\boldsymbol{n} \boldsymbol{b}}^{*}\right)$ is corrected [15] to account for the pressure gradient

$$
\boldsymbol{u}_{f}=\boldsymbol{u}_{f}^{*}-\Delta t(\nabla p)_{f}
$$

If the corrected face velocity is used to compute the corrected mass flux $\left(F_{f}^{n+1}=\boldsymbol{u}_{\boldsymbol{f}} \cdot \boldsymbol{S}_{\boldsymbol{f}}\right)$, then Eq. (8) gives a pressure Poisson equation

$\sum_{f}\left[\boldsymbol{u}_{f}^{*}-\Delta t(\nabla p)_{f}\right] \cdot \boldsymbol{S}_{\boldsymbol{f}}=0 \Longrightarrow \sum_{f}(\nabla p)_{f} \cdot \boldsymbol{S}_{\boldsymbol{f}}=\frac{1}{\Delta t} \sum_{f} F_{f}^{*}$,

where $\left(F_{f}^{*}=\boldsymbol{u}_{f}^{*} \cdot \boldsymbol{S}_{f}\right)$ is the provisional mass flux. Pressure field computed from Eq. (12) is used in Eq. (9) to obtain the correct velocity $\left(\boldsymbol{u}^{n+\boldsymbol{l}}\right)$.

\subsection{Linear solvers}

Numerical approximations for the flow variables $(\phi)$ result in systems of linear equations at the fluid $\left(A_{F} \Phi=b_{F}\right)$ and ghost $\left(A_{G} \Phi=b_{G}\right)$ cells. These two sets are solved simultaneously so that the progressive changes in the ghost point values implicitly force the boundary conditions. The velocity equation being a diagonally dominant system converges fast, while the pressure Poisson equation needs to be preconditioned in order to accelerate its convergence. In the present work, Stone's strongly implicit procedure SIP [16] is used to precondition the BiCGSTAB technique [17].

\subsection{Computation of the fluid forces on the moving boundary}

If a control volume (having volume $V$ and surface area $S$ ) encloses the body, then force in the $i$ th direction using the momentum balance principle is

$$
F_{i}=\int_{V} \frac{\partial\left(\rho u_{i}\right)}{\partial t} d V+\int_{S} \rho u_{i}(\boldsymbol{u} \cdot \hat{\boldsymbol{n}} d S)+\int_{S} p\left(\hat{\boldsymbol{n}_{\boldsymbol{i}}} \cdot \hat{\boldsymbol{n}} d S\right)+\int_{V} \rho a_{i} d V
$$

$\hat{\boldsymbol{n}}$ and $\hat{\boldsymbol{n}}_{i}$ are unit vectors along the surface normal of the chosen control volume and $i$ th direction, respectively.

All the computations, in the present work, were performed using nine processors of an Intel Xeon E5620 cluster loaded with MPI libraries.

\section{Uniform flow past a stationary sphere}

The wake behind a sphere is marked by several regimes at increasing $R e$. Creeping flow in which the flow divides, swims over the surface and reunites at the rear end of the sphere exists for $R e \rightarrow 0$. The laminar boundary layer separates at $R e \approx 20$ from the sphere surface leading to a laminar wake. Four flow regimes of laminar wake are steady axisymmetric $(20<R e<210)$, steady planar symmetric $(210<R e<270)$, unsteady planar symmetric $(280<\operatorname{Re}<420)$ and unsteady asymmetric $(420<\operatorname{Re}<800)$.

First regime is characterized with an axisymmetric stationary vortex-ring attached at the rear end of the sphere. In the second regime, the axisymmetry is replaced by a planar symmetry. A trail of two parallel counter rotating vortices is observed, and is known as the double-threaded wake. The third regime is characterized by the periodic vortex shedding, where vortices are shed as interconnected vortex loops; maintaining a planar symmetry about a plane passing through the wake centerline. In the fourth regime, irregular and asymmetric shedding of hairpin vortices is observed.

\subsection{Computational details}

Figure 2 shows schematic diagram describing the problem, computational domain and boundary conditions employed. Three-dimensional numerical simulations were carried out on a non-uniform cartesian grid $217 \times 181 \times 181$ in the stream-wise $(x)$, lateral $(y)$ and side directions $(z)$, respectively. A cubical domain of edge 3 with grid size $151 \times$ $151 \times 151$ was used to provide a uniform finer mesh engulfing the sphere which generated grid resolution $\Delta x=$ $\Delta y=\Delta z=0.02$ in and around the sphere. Computations were performed for $R e=100,250$ and 300 with time increment $\Delta t=0.003$. 


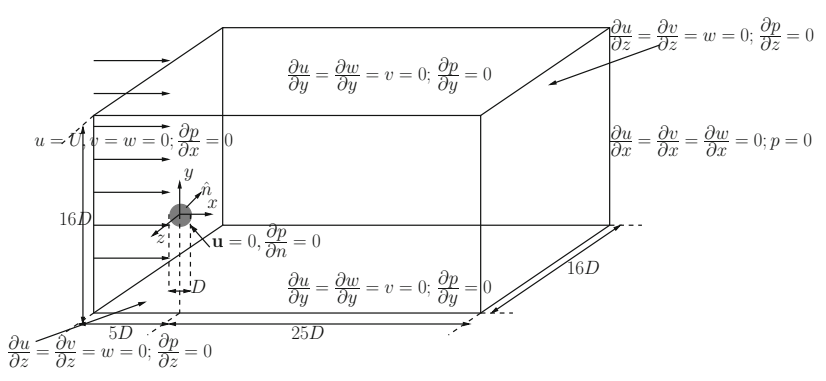

Figure 2. Schematic diagram for uniform flow past a stationary sphere.

\subsection{Steady axisymmetric flow, $R e=100$}

The coefficient of lateral force $\left(C_{L}\right)$ and coefficient of sidewise force $\left(C_{S}\right)$ are asymptotically zero. The coefficient of drag $\left(C_{D}=1.085\right)$ is in good agreement to the reported [19] value of 1.080 The wake structure [18] using $Q=$ 0.001 (see figure 3 top row) reveals the axisymmetric vortex-ring at $R e=100$. The $z$-component of vorticity shown in figure 4 agrees well with the reported [19] trend, and is similar to the steady near wake of a circular cylinder at subcritical Reynolds numbers.

\subsection{Steady planar symmetric flow, $R e=250$}

The wake remains steady with $C_{D}=0.702$, in agreement to the reported [19] value of 0.700 . The non-zero $C_{L}=-0.066$, compared to -0.062 reported by Johnson and Patel [19], signifies the loss of axial symmetry. Instead
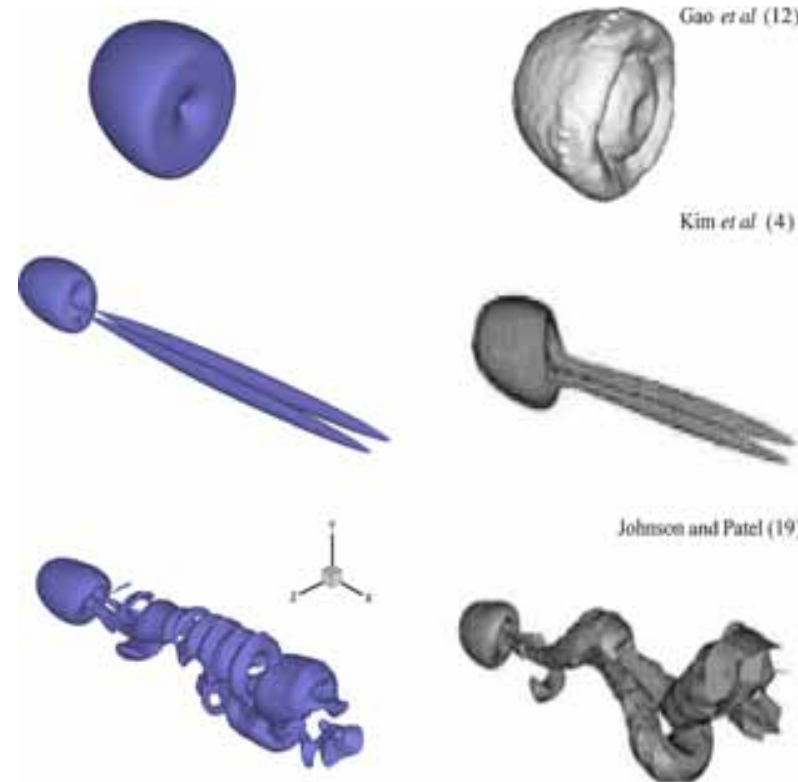

Johnsee and Patel (19)

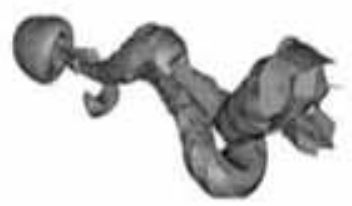

Figure 3. Comparison of computed wake structures [18] with reported results for flow past a sphere at $R e=100,250$ and 300 . the flow admits a planar symmetry $(x, y)$-plane at $z=0$, which is supported by zero $C_{S}$. The wake structure (see figure 3 middle row) demonstrates the trail of two counterrotating vortices, which in literature is called a doublethreaded wake. At this $R e$ the flow acquires three-dimensionality, which is evident by the three components of vorticity (see figure 5) in agreement with the reported results [19]. Stream-wise vorticity $\left(w_{x}\right)$ contours in (figure 5 top row) reveal an antisymmetric distribution about the symmetry plane. As the flow has a planar symmetry; the lateral vorticity $\left(w_{y}\right)$ in (figure 5 middle row) is symmetric, while the side-wise vorticity $\left(w_{z}\right)$ appears to be shifted towards positive- $y$ in (figure 5 bottom row).

\subsection{Unsteady planar symmetric flow, $R e=300$}

At $R e=300$ the periodic signals of $C_{D}$ and $C_{L}$ indicate flapping of the wake, which is seen at a much lower Reynolds number for a circular cylinder wake. However, the zero coefficient of side-wise force results from the prevailing planar symmetry observed at $R e=250$. The wake structure (see figure 3 bottom row) consists of hairpin like inter-connected vortex loops. Table 1 compares the global flow parameters: average coefficient of drag $\left(<C_{D}>\right)$, average coefficient of lateral force $\left(\left\langle C_{L}\right\rangle\right)$ and Strouhal number $(S t)$ with the literature. Figure 6 shows the instantaneous vorticity contours at three different planes, which agree well with the reported results [19].

\section{Forced transverse oscillation of a circular cylinder}

Den Hartog [20] was the first to report synchronization of the periodic wake behind a transversely oscillating cylinder. Within the synchronization, jumps in the phase and amplitude of the lift and drag forces were reported by Bishop and Hassan [21]. Vibration of the cylinder at the upper and lower frequency limits of the synchronized regime causes vortex street to be compressed and expanded [22], respectively. The oscillating cylinder imposes timing and frequency of vortex shedding on the wake in the synchronized regime [23]. Williamson and Roshko [24] suggested that the jumps [21] were associated with the change of vortex shedding modes namely $2 \mathrm{~S}$ and $2 \mathrm{P}$.

\subsection{Computational details}

Figure 7 shows schematic diagram describing the problem, computational domain and boundary conditions employed. Two-dimensional numerical simulations were carried out on a non-uniform cartesian grid $293 \times 226$ in the streamwise $(x)$ and transverse (y) directions, respectively. A square domain of side 1.2 with grid size $161 \times 161$ was 


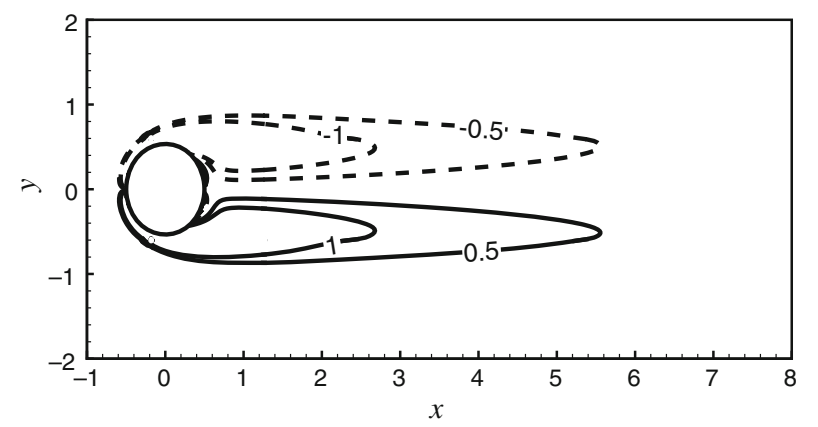

Figure 4. Vorticity contour ( $z$-component) for flow past a sphere at $R e=100$.
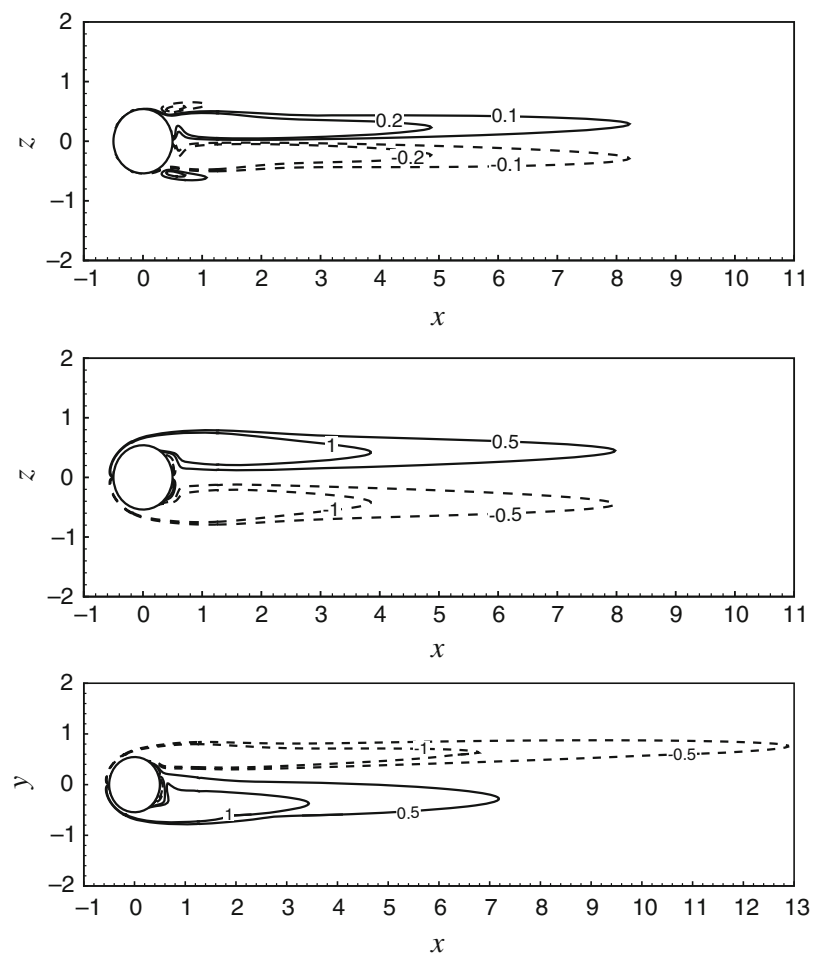

Figure 5. Vorticity contours for flow past a sphere at $R e=250$.

Table 1. Comparison of computed global flow parameters for flow past a sphere at $R e=300$ with reported results.

\begin{tabular}{lccc}
\hline Work & $\left\langle C_{D}\right\rangle$ & $\left\langle C_{L}\right\rangle$ & $S t$ \\
\hline Present & 0.659 & -0.069 & 0.137 \\
Johnson and Patel [19] & 0.656 & -0.069 & 0.137 \\
Kim et al [4] & 0.657 & 0.067 & 0.134 \\
\hline
\end{tabular}

used to provide a uniform finer mesh engulfing the cylinder which generated grid resolution $\Delta x=\Delta y=0.007$ in and around the cylinder. We defined a frequency ratio $\left(f_{R}=f_{e} / f_{o}\right)$, where $f_{o}$ is the vortex shedding frequency from the stationary cylinder. If $S t_{o}=f_{o} D / U$ is the Strouhal
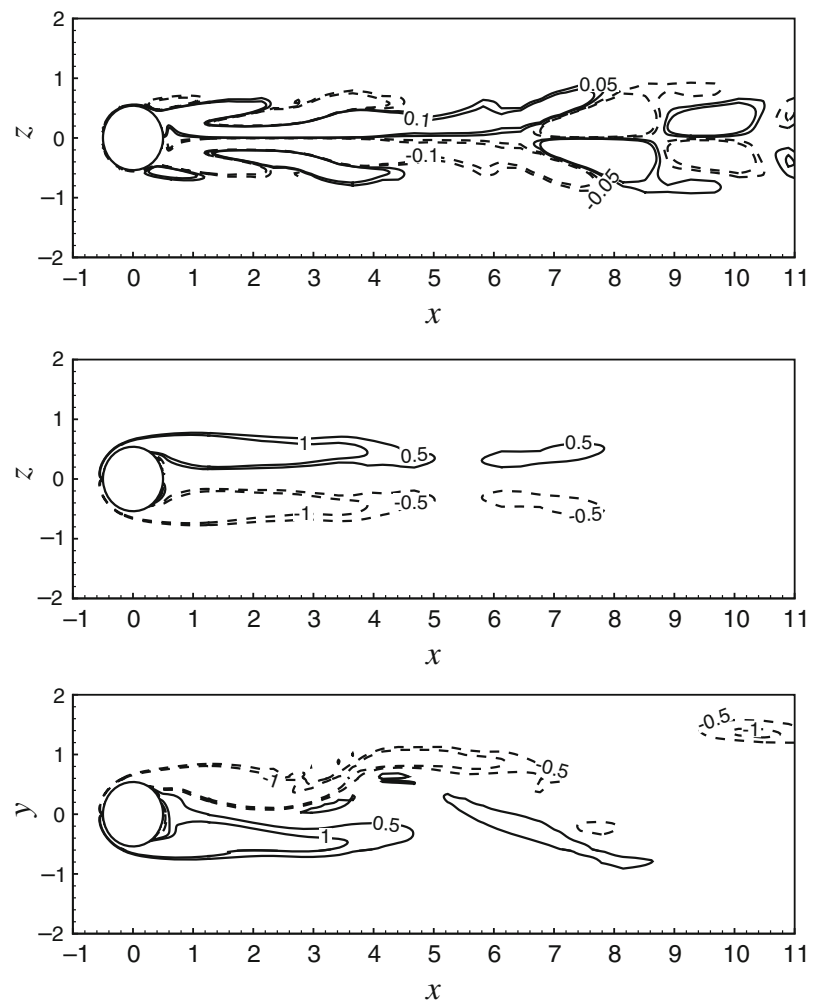

Figure 6. Vorticity contours for flow past a sphere at $R e=300$.

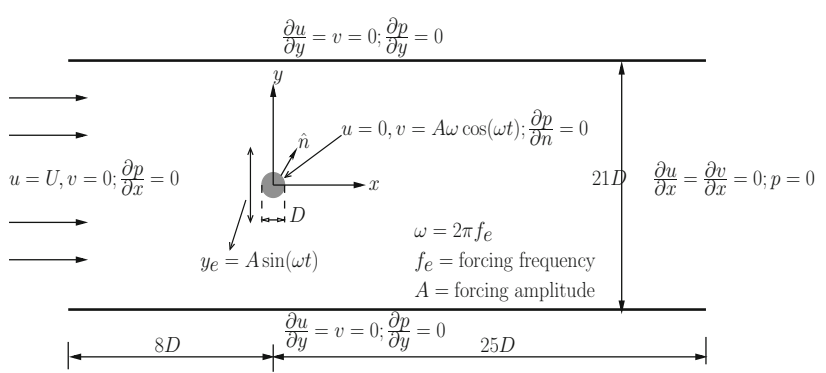

Figure 7. Schematic diagram for forced transverse oscillation of a circular cylinder.

number associated with the vortex shedding from a stationary cylinder, then the forced Strouhal number $\left(S t_{e}=f_{e} D / U\right)$ can be expressed as $S t_{e}=f_{R} S t_{o}$; in this study $S t_{o}$ was found out to be 0.201 . Computations were performed for non-dimensional amplitude $(A=0.2), R e=$ 185 and $0.5 \leq f_{R} \leq 2.0$ with time increment $(\Delta t=0.0004)$.

\subsection{Results}

Figure 8 shows that the time evolution of the coefficient of drag $\left(C_{D}\right.$, upper signals) and coefficient of lift $\left(C_{L}\right.$, lower signals) match well with the reported [25] results. The signals for $f_{R}=0.8,0.9$ and 1.0 are purely sinusoidal, while the signals for $f_{R}=1.1,1.12$ and 1.2 are complex wave-forms. 

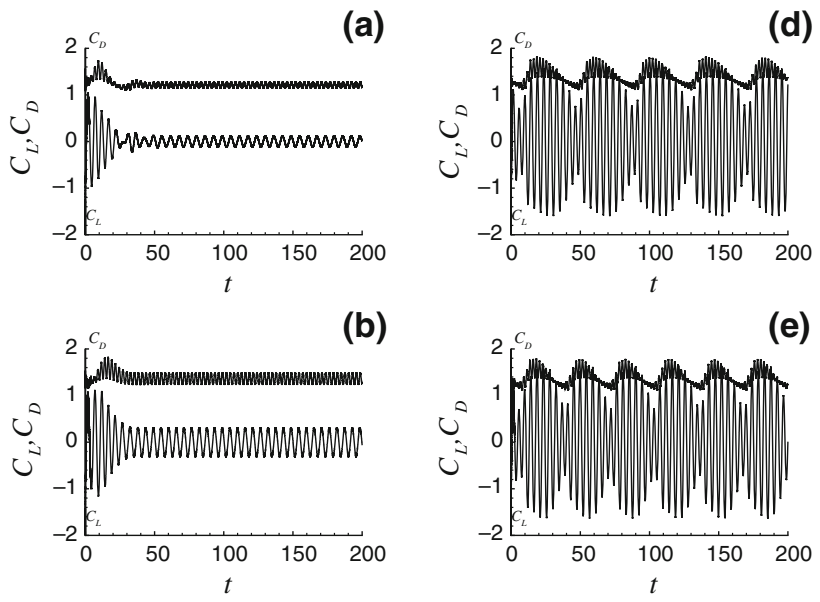

(c)
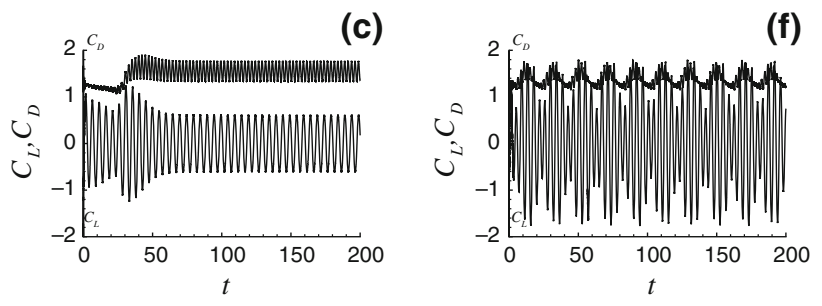

Figure 8. Time evolution of the coefficient of drag and lift for forced transverse oscillation of a circular cylinder, (a) $f_{R}=0.8$, (b) $f_{R}=0.9,(\mathbf{c}) f_{R}=1.0,(\mathbf{d}) f_{R}=1.1,(\mathbf{e}) f_{R}=1.12$, (f) $f_{R}=1.2$.

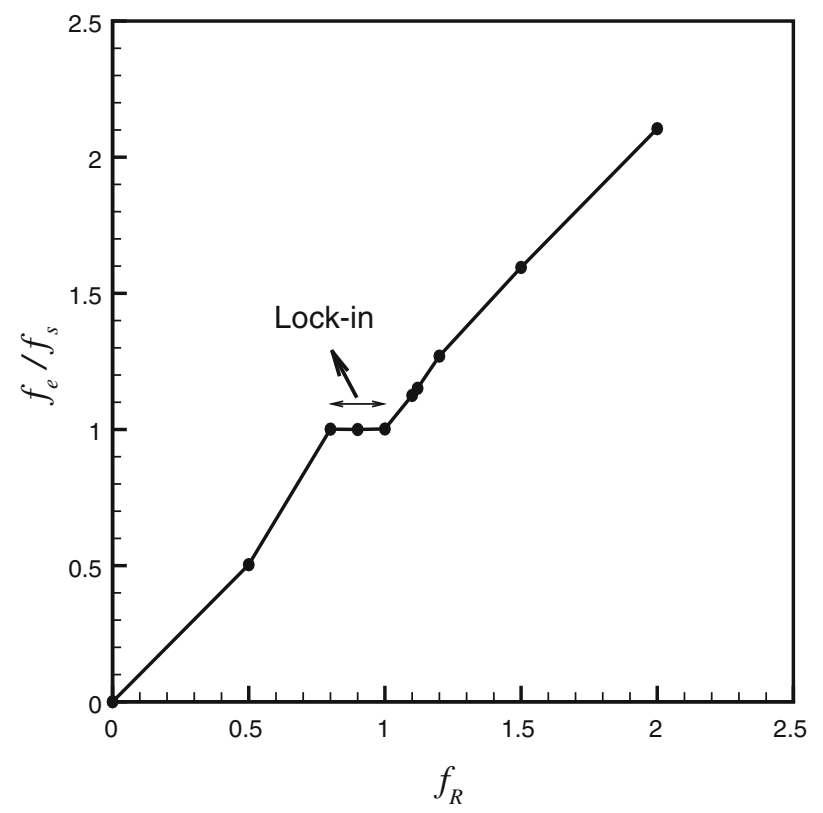

Figure 9. Ratio of the forcing and the vortex shedding frequency as a function of $f_{R}$ for forced transverse oscillation of a circular cylinder.

The complex wave-forms reflect [21] the existence of two frequencies: (1) the natural vortex shedding frequency $\left(f_{o}\right)$ of a stationary circular cylinder and (2) the forcing frequency $\left(f_{e}\right)$. Purely sinusoidal signals are consequence of the lock-in

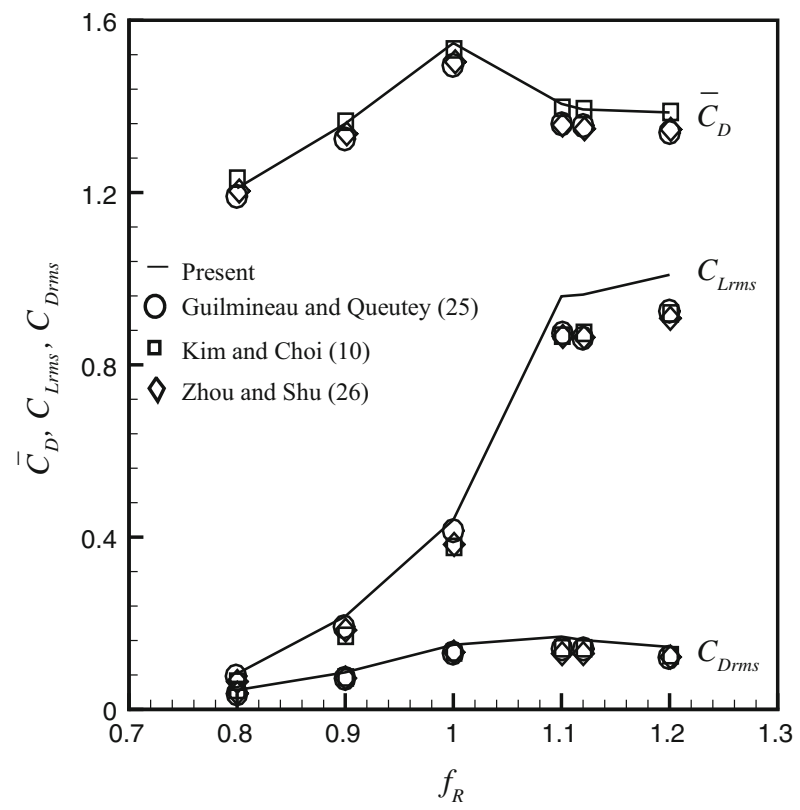

Figure 10. Comparison of computed global flow parameters for forced transverse oscillation of a circular cylinder with reported results.

state of the wake $\left(0.8 \leq f_{R} \leq 1.0\right)$, where the ratio of the forcing frequency to the vortex shedding frequency is unity. Outside the lock-in regime the dominant vortex shedding frequency $\left(f_{s}\right)$ varies almost linearly with $f_{R}$ (see figure 9). The global parameters: (i) average coefficient of drag $\left(\bar{C}_{D}\right)$, (ii) root mean square (rms) value of the coefficient of lift $\left(C_{\text {Lrms }}\right)$ and (iii) rms value of the coefficient of drag $\left(C_{D r m s}\right)$ shown in figure 10 are in good agreement with the reported results of Guilmineau and Queutey [25], Kim and Choi [10], and Zhou and Shu [26].

\section{Flow past a rotating circular cylinder in a uniform flow}

The rotation of a circular cylinder in a uniform flow is expected to reduce the flow-induced oscillations [27]. In addition to $R e=U D / v$ the other parameter that influences the flow is non-dimensional rotation rate $(\alpha=D \omega / 2 U)$, where $\omega$ represents the angular velocity of the cylinder about its axis. Kang et al [27] found that the vortex shedding is completely suppressed beyond a critical non-dimensional rotational speed $\left(\alpha_{L}\right)$, which increases logarithmically with $R e$. In a narrow range, $4.8<\alpha<5.15$ at $R e=100$ Stojkovic et al [28] interestingly found again an unsteady behavior in the flow field with large amplitudes in the fluctuation of the drag and lift forces. At $R e=200$ and $4.34 \leq \alpha \leq 4.7$ a reappearance of unsteady wake was reported by Mittal and Kumar [29] which was later experimentally confirmed by Kumar et al [30]. 


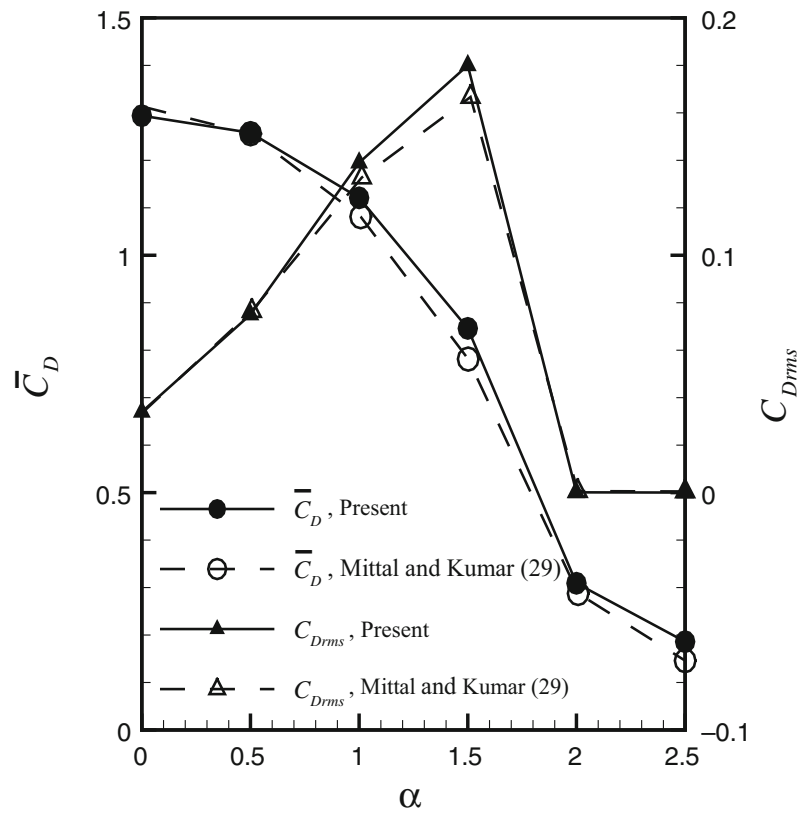

Figure 11. Comparison of the average and rms values of the drag coefficient for rotating circular cylinder with reported results.

\subsection{Computational details}

All the computational features employed were the same as that of the previous section. Two-dimensional simulations were performed at $R e=200$ and $(0 \leq \alpha \leq 2.5)$ in steps of 0.5 .

\subsection{Results}

Figure 11 shows that $\bar{C}_{D}$ decreases while $C_{\text {Drms }}$ increases up to $\alpha \leq 1.5$ for which the vortex shedding is observed. Figure 12 shows that $\bar{C}_{L}$ increases while $C_{\text {Lrms }}$ is nearly constant for $\alpha \leq 1.5$. In agreement to Mittal and Kumar [29] our results show no vortex shedding for $\alpha=2$ and 2.5, which is supported by zero $C_{D r m s}$ and $C_{\text {Lrms }}$. Moreover, $\left|\bar{C}_{L}\right|$ continuously increases with $\alpha$ because of the Magnus effect.

\section{Conclusions}

The present work has extended the previously proposed IDW technique for reconstruction on the immersed boundaries to accommodate zero-gradient pressure situation. The methodology is applied to three-dimensional flows at moderate Reynolds number by employing MPIbased parallelization. Non-inertial frame of reference is introduced to deal with moving boundary problems which reportedly have produced spurious force oscillations in the inertial frame of reference. The developed method is tested

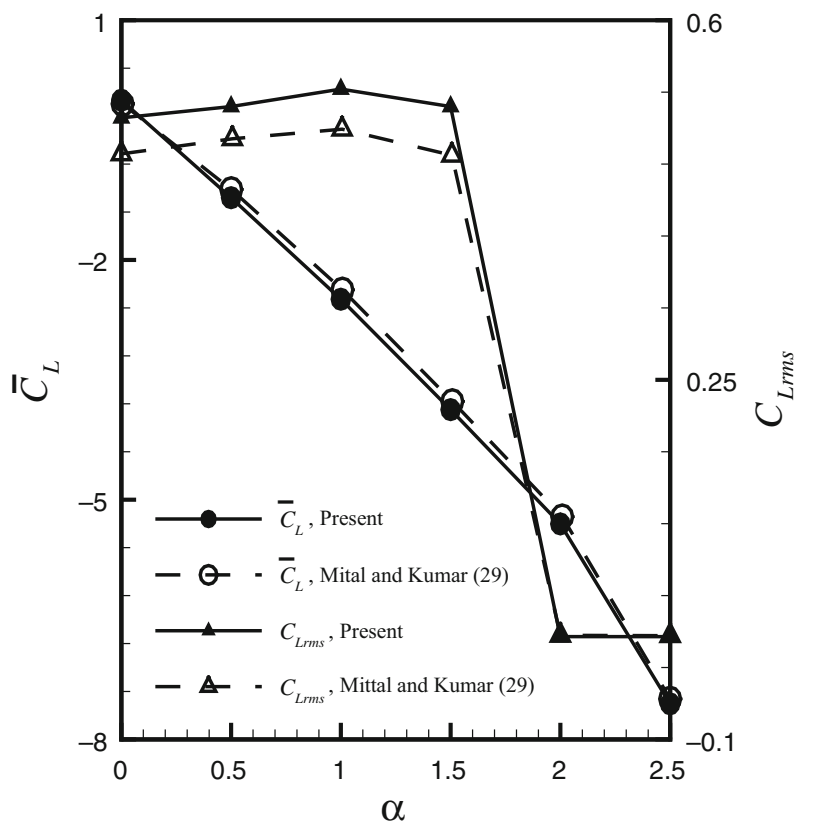

Figure 12. Comparison of the average and rms values of the lift coefficient for rotating circular cylinder with reported results.

on flows past transversely oscillating and rotating cylinders. The results thus obtained compare well with the reported trends.

\section{Acknowledgments}

The present research was carried out on the funds available through the institute start-up Grant SG/ME/P/ARKD/1/ 2009-2010 and DST First Track Grant SERC/ET-0166/ 2011.

\section{Appendix}

Finite difference approximations of the partial derivatives in non-uniform grids at a point $\left(x_{i}, y_{j}, z_{k}\right)$

First derivatives

$$
\begin{aligned}
\frac{\partial \phi_{i}}{\partial x}= & \frac{\phi_{i+1}\left(\Delta x_{i-1}\right)^{2}-\phi_{i-1}\left(\Delta x_{i+1}\right)^{2}-\phi_{i}\left(\left(\Delta x_{i-1}\right)^{2}-\left(\Delta x_{i+1}\right)^{2}\right)}{\Delta x_{i+1} \Delta x_{i-1}\left(\Delta x_{i+1}+\Delta x_{i-1}\right)} \\
& +O\left[(\Delta x)^{2}\right] \\
\frac{\partial \phi_{j}}{\partial y}= & \frac{\phi_{j+1}\left(\Delta y_{j-1}\right)^{2}-\phi_{j-1}\left(\Delta y_{j+1}\right)^{2}-\phi_{j}\left(\left(\Delta y_{j-1}\right)^{2}-\left(\Delta y_{j+1}\right)^{2}\right)}{\Delta y_{j+1} \Delta y_{j-1}\left(\Delta y_{j+1}+\Delta y_{j-1}\right)} \\
& +O\left[(\Delta y)^{2}\right]
\end{aligned}
$$




$$
\begin{aligned}
\frac{\partial \phi_{k}}{\partial z}= & \frac{\phi_{k+1}\left(\Delta z_{k-1}\right)^{2}-\phi_{k-1}\left(\Delta z_{k+1}\right)^{2}-\phi_{k}\left(\left(\Delta z_{k-1}\right)^{2}-\left(\Delta z_{k+1}\right)^{2}\right)}{\Delta z_{k+1} \Delta z_{k-1}\left(\Delta z_{k+1}+\Delta z_{k-1}\right)} \\
& +O\left[(\Delta z)^{2}\right] .
\end{aligned}
$$

Second derivatives

$$
\begin{aligned}
\frac{\partial^{2} \phi_{i}}{\partial x^{2}}= & 2\left(\frac{\phi_{i+1} \Delta x_{i-1}+\phi_{i-1} \Delta x_{i+1}-\phi_{i}\left(\Delta x_{i+1}+\Delta x_{i-1}\right)}{\Delta x_{i+1} \Delta x_{i-1}\left(\Delta x_{i+1}+\Delta x_{i-1}\right)}\right) \\
& +O[\Delta x]
\end{aligned}
$$

$$
\begin{aligned}
\frac{\partial^{2} \phi_{j}}{\partial y^{2}}= & 2\left(\frac{\phi_{j+1} \Delta y_{j-1}+\phi_{j-1} \Delta y_{j+1}-\phi_{j}\left(\Delta y_{j+1}+\Delta y_{j-1}\right)}{\Delta y_{j+1} \Delta y_{j-1}\left(\Delta y_{j+1}+\Delta y_{j-1}\right)}\right) \\
& +O[\Delta y]
\end{aligned}
$$

$$
\begin{aligned}
\frac{\partial^{2} \phi_{k}}{\partial z^{2}}= & 2\left(\frac{\phi_{k+1} \Delta z_{k-1}+\phi_{k-1} \Delta z_{k+1}-\phi_{k}\left(\Delta z_{k+1}+\Delta z_{k-1}\right)}{\Delta z_{k+1} \Delta z_{k-1}\left(\Delta z_{k+1}+\Delta z_{k-1}\right)}\right) \\
& +O[\Delta z] .
\end{aligned}
$$

Mixed derivatives

$\frac{\partial^{2} \phi_{i, j}}{\partial x \partial y}=\frac{\phi_{i+1, j+1}-\phi_{i+1, j-1}-\phi_{i-1, j+1}+\phi_{i-1, j-1}}{\left(\Delta x_{i+1}+\Delta x_{i-1}\right)\left(\Delta y_{j+1}+\Delta y_{j-1}\right)}+O[\Delta x, \Delta y]$

$\frac{\partial^{2} \phi_{i, k}}{\partial x \partial z}=\frac{\phi_{i+1, k+1}-\phi_{i+1, k-1}-\phi_{i-1, k+1}+\phi_{i-1, k-1}}{\left(\Delta x_{i+1}+\Delta x_{i-1}\right)\left(\Delta z_{k+1}+\Delta z_{k-1}\right)}+O[\Delta x, \Delta z]$

$\frac{\partial^{2} \phi_{j, k}}{\partial y \partial z}=\frac{\phi_{j+1, k+1}-\phi_{j+1, k-1}-\phi_{j-1, k+1}+\phi_{j-1, k-1}}{\left(\Delta y_{j+1}+\Delta y_{j-1}\right)\left(\Delta z_{k+1}+\Delta z_{k-1}\right)}+O[\Delta y, \Delta z]$,

where

$$
\begin{aligned}
& \Delta x_{i+1}=x_{i+1}-x_{i}, \Delta x_{i-1}=x_{i}-x_{i-1}, \Delta y_{j+1}=y_{j+1}-y_{j} \\
& \Delta y_{j-1}=y_{j}-y_{j-1}, \Delta z_{k+1}=z_{k+1}-z_{k}, \Delta z_{k-1}=z_{k}-z_{k-1} .
\end{aligned}
$$

\section{References}

[1] Peskin C S 1972 Flow patterns around heart valves:a numerical method. J. Comput. Phys. 10: 252-271

[2] Goldstein D, Handler R and Sirovich L 1993 Modelling a noslip flow boundary with an external force field. J. Comput. Phys. 105: 354-366

[3] Mohd-Yosuf J 1997 Combined immersed-boundary/ b-spline methods for simulations of flow in complex geometries. CTR, Annual Research Briefs 317-327
[4] Kim J, Kim D and Choi H 2001 An immersed-boundary finite-volume method for simulations of flow in complex geometries. J. Comput. Phys. 171: 132-150

[5] Majumdar S, Iaccarino G and Durbin P 2001 Rans solvers with adaptive structured boundary non-conforming grids. CTR, Annual Research Briefs 353-366

[6] Tseng Y H and Ferziger J H 2003 A ghost-cell immersed boundary method for flow in complex geometry. J. Comput. Phys. 192: 593-623

[7] Mittal R, Dong H, Bozkurttas M, Najjar F M, Vargas A and Loebbecke A V 2008 A versatile sharp interface immersed boundary method for incompressible flows with complex boundaries. J. Comput. Phys. 227: 4825-4852

[8] De A K 2014 An implicit non-staggered cartesian grid method for incompressible viscous flows in complex geometries. Sadhana Ind. Acad. Sci. 39: 1071-1094

[9] Lee J and You D 2013 An implicit ghost-cell immersed boundary method for simulations of moving body problems with control of spurious force oscillations. J. Comput. Phys. 233: 295-314

[10] Kim D and Choi H 2006 Immersed boundary method for flow around an arbitrarily moving body. J. Comput. Phys. 212: $662-680$

[11] Iaccarino G and Verzicco R 2003 Immersed boundary technique for turbulent flow simulations. Appl. Mech. Rev. 56: $331-347$

[12] Gao T, Tseng Y H and Lu X Y 2007 An improved hybrid cartesian/immersed boundary method for fluid-solid flows. Int. J. Numer. Meth. Fluids 55: 1189-1211

[13] Mittal R and Iaccarino G 2005 Immersed boundary methods. Annu. Rev. Fluid Mech. 37: 239-261

[14] Pope S B 2000 Turbulent lows. Cambridge University Press. Cambridge

[15] De A K and Dalal A 2006 Numerical simulation of unconfined flow past a triangular cylinder. Int. J. Numer. Meth. Fluids 52: 801-821

[16] Stone H L 1968 Iterative solution of implicit approximation of multidimensional partial differential equations. SIAM J. Numer. Anal. 5: 530-558

[17] Zhang S-L 1997 Gpbi-cg:Generalized product-type methods based on bi-cg for solving nonsymmetric linear systems. SIAM J. Sci. Comput. 18(2): 537-551

[18] Hunt J C R, Wray A A and Moin P 1988 Eddies, streams, and convergence zones in turbulent flows. Center for Turbulence Research Report CTR-S88 2, 193-208

[19] Johnson T A and Patel V C 1999 Flow past a sphere up to a Reynolds number of 300. J. Fluid Mech. 378: 19-70

[20] Den Hartog J P 1934 The vibration problems in engineering. In: Proceedings of the 4th International Congresson Applied Mechanics Cambridge, U K, pp. 36-53

[21] Bishop R E D and Hassan A Y 1964 The lift and drag forces on a circular cylinder oscillating in a flowing fluid. Proc. R. Soc. Lond. Ser. A 277: 51-75

[22] Koopmann G H 1967 The vortex wakes of vibrating cylinders at low reynolds number. J. Fluid Mech. 23: 501-512

[23] Zdravkovich M M 1982 Modification of vortex shedding in the synchronization range. ASME J. Fluids Eng. 104: 513-517

[24] Williamson C H K and Roshko A 1988 Vortex formation in the wake of an oscillating cylinder. J. Fluids Struct. 2: 355-381 
[25] Guilmineau E and Queutey P 2002 A numerical simulation of vortex shedding from an oscillating circular cylinder. $J$. Fluids Struct. 16: 773-794

[26] Zhou C H and Shu C 2011 A local domain-free discretization method for simulation of incompressible flows over moving bodies. Int. J. Numer. Meth. Fluids 66: 162-182

[27] Kang S, Choi H and Lee S 1999 Laminar flow past a rotating circular cylinder. Phys. Fluids 11: 3312-3321
[28] Stojkovic D, Breuer M and Durst F 2002 Effect of high rotation rates on the laminar flow around a circular cylinder. Phys. Fluids 14: 3160-3178

[29] Mittal S and Kumar B 2003 Flow past a rotating cylinder. $J$. Fluid Mech. 476: 303-334

[30] Kumar S, Cantu C and Gonzalez B 2011 Flow past a rotating cylinder at low and high rotation rates. ASME J. Fluid Eng. 133: 041201-1-041201-9 